\title{
A three-year longitudinal study of healthy lifestyle behaviors and adherence to pharmacological treatments in newly diagnosed patients with acute coronary syndrome: hierarchical linear modeling analyses
}

\author{
Andrea Greco $^{1}$ (D) $\cdot$ Agostino Brugnera $^{1} \cdot$ Marco D'Addario $^{2} \cdot$ Angelo Compare $^{1} \cdot$ Cristina Franzelli $^{3}$. \\ Alessandro Maloberti $^{4,5} \cdot$ Cristina Giannattasio $^{4,5} \cdot$ Francesco Fattirolli $^{6,7} \cdot$ Patrizia Steca $^{2}$
}

Received: 30 March 2020 / Accepted: 8 July 2020 / Published online: 1 August 2020

(C) The Author(s) 2020

\begin{abstract}
Aim Healthy lifestyle behaviors and a good adherence to pharmacological treatments are important predictors of lower recurrence rates and better overall outcomes among patients with an established acute coronary syndrome (ACS). The present study sought to investigate the longitudinal trajectories of these behaviors years after the onset of an ACS.

Subject and methods We recruited a sample of 275 newly diagnosed consecutive patients at their first ACS event (mean age: $57.1 \pm 7.87$ years; $84 \%$ males) admitted to a cardiac rehabilitation program from three large public hospitals in Northern Italy. Patients completed a battery of sociodemographic questionnaires, which evaluated healthy lifestyles (smoking status, alcohol intake, diet, and physical activity) and adherence to pharmacological treatments, at five time-points (pre-event, 6-, 12-, 24-, and 36-month follow-ups). Longitudinal trajectories were examined through hierarchical (generalized) linear models, controlling for several demographic and clinical variables.

Results We found significant changes in all healthy lifestyles from pre-event to the 6-month follow-up, suggesting the adoption of healthier behaviors soon after the cardiac event. However, from the 6-month up to the 3-year follow-up, patients experienced small but significant declines in their self-reported levels of healthy dietary behaviors and physical activity. Further, we found that the odds of being at medium risk of non-adherence to the pharmacological treatments significantly increased over the course of 3 years.

Conclusion Given the negative long-term trajectories in specific lifestyles and adherence to pharmacological treatments, cardiac rehabilitation programs are suggested to provide repeated psychological interventions aimed at fostering patients' capabilities to self-regulate their habitual behaviors.
\end{abstract}

Keywords Acute coronary syndrome $\cdot$ Healthy behaviors $\cdot$ Adherence to pharmacological treatments $\cdot$ Diet $\cdot$ Physical activity

Acute coronary syndrome (ACS) is one of the most frequent causes of hospitalization in developed countries (Piepoli et al. 2016), and it is associated with high rates of morbidity, mortality, and a substantial economic and psychosocial burden

Andrea Greco and Agostino Brugnera are co-first authors.

Andrea Greco

andrea.greco@unibg.it

1 Department of Human and Social Sciences, University of Bergamo, P.le S. Agostino, 2, 24129 Bergamo, Italy

2 Department of Psychology, University of Milan-Bicocca, Milan, Italy

3 Cardiac Rehabilitation Centre, Istituti Clinici di Perfezionamento Hospital, Milan, Italy
(Nabel and Braunwald 2012; Piepoli et al. 2016; Ruff and Braunwald 2010). Patients with ACS also have a higher risk of recurrent cardiovascular events (Piepoli et al. 2016), and thus their clinical management is challenging. Modifiable risk
4 School of Medicine and Surgery, University of Milano-Bicocca, Milan, Italy

5 Cardiology 4, “A. De Gasperis” Department, ASST GOM Niguarda Ca' Granda Hospital, Milan, Italy

6 Department of Medical and Surgical Critical Care, Cardiac Rehabilitation Unit, University of Florence, Florence, Italy

7 Azienda Ospedaliero-Universitaria Careggi, Florence, Italy 
factors (regardless of gender, ethnicity or geographic origin) account for more than $90 \%$ of the risk of an initial acute myocardial infarction (regardless of gender, ethnicity or geographic origin; ESC Scientific Document Group et al. 2017; Yusuf et al. 2004), suggesting that ACS is a largely preventable disorder (ESC Scientific Document Group et al. 2017; Nabel and Braunwald 2012; Yusuf et al. 2004).

Some of the most important risk factors are individual lifestyle (i.e., smoking, alcohol intake, unhealthy dietary habits, and physical inactivity), which accounts for between $12.5 \%$ and $26.4 \%$ of population-attributable risk (Yusuf et al. 2004). Most of these adverse effects are reversible, with cardiovascular risk decreasing substantially once a positive, stable lifestyle change is reached (e.g., within the first 2 years of smoking cessation, or with at least $30 \mathrm{~min}$ of moderate to vigorous aerobic exercise five times a week; Erhardt 2009; Piepoli et al. 2016). This suggests that the management of lifestyle behaviors is a highly cost-effective intervention in secondary prevention and cardiac rehabilitation settings, which could effectively improve the prognosis of adherent patients by reducing morbidity, mortality, and the risk of recurrent events (Chow et al. 2010; Fattirolli et al. 2018; Ford et al. 2007; Ibanez et al. 2017; Peersen et al. 2017; Piepoli et al. 2016). Indeed, the guidelines on cardiovascular disease prevention of the Joint European Societies (JES) of Cardiology suggest that rehabilitation programs for individuals with ACS should (i) focus on lifestyle recommendations and (ii) promote a strict adherence to the pharmacological treatment (e.g., daily assumption of anti-platelets, statins, and beta-blockers; Piepoli et al. 2016). However, less than $50 \%$ of new AMI cases are prescribed with appropriate secondary prevention treatments at discharge (Tea et al. 2018), while the compliance to these regimens tends to decline within 6 months of discharge from hospital (Chow et al. 2010; Kotseva et al. 2019; Tang et al. 2013). In addition, a recent study evidenced that both patients with a history of heart disease or newly diagnosed ones have poor knowledge and awareness of their cardiovascular risk factors, suggesting the need of improving patients' understanding of the disease and their compliance to a cardioprotective lifestyle (Wójcicki et al. 2020).

To date, the long-term data on these outcomes are missing, and therefore the trajectories of healthy behaviors years after the onset of an ACS remain unknown. Given the clinically significant impact that evidence on this topic would provide, further studies are warranted. Therefore, in the current study we sought to investigate longitudinal trajectories and associated baseline predictors of (i) adherence to pharmacological treatments, and (ii) four unhealthy lifestyle behaviors (i.e., tobacco use, alcohol intake, unhealthy dietary habits, and physical inactivity, which are comprised among the cardiovascular health metrics of the American Heart Association; Lloyd-Jones Donald et al. 2010), in a cohort of 275 consecutive ACS patients at their first coronary event. These patients are in an awkward situation since they are at a point in their lives where they have to face a shocking event and receive multiple demands to change their lifestyles. A deeper knowledge on how pharmacological adherence and health relayed behaviors develop over time may help to personalize early interventions of secondary prevention, making them more effective. Participants were assessed at 5 time-points, namely at baseline (i.e., soon after discharge from coronary care units and at the beginning of cardiac rehabilitation (CR) programs, where they retrospectively reported information on their behaviors before the onset of the cardiac event), 6 months, and at 1, 2, and 3 years after the cardiovascular event. We modeled the individual rate of change from pre-event over the course of 3 years follow-up through an advanced statistical technique, namely hierarchical linear models (HLMs) with a piecewise regression approach. We tested two hypotheses. First, we examined if patients would experience longitudinal decreases in adherence to pharmacological treatments, from the 6-month to the 3-year follow-up, as suggested by previous studies that followed cardiovascular patients for 12 months (e.g., Crowley et al. 2015; Molloy et al. 2014). Second, we examined if patients would experience strong increases in the four lifestyle behaviors from pre-event to 6-months after, and small but significant decreases from the 6-month to the 3-year follow-up, as suggested by previous studies that followed patients for 6 or 12 months (e.g., Chow et al. 2010; Kotseva et al. 2019; Steca et al. 2017; Tang et al. 2013). In essence, we expect to replicate findings from previous longitudinal studies in a much longer and unexplored period of time than previously. In both hypotheses, we examined the extent to which baseline patient characteristics were associated with these trajectories, thus adjusting all analyses for potential sociodemographic (i.e., age, sex, educational level, working, living, and marital status) and clinical confounders (i.e., family history, and a diagnosis of hypertension, diabetes, dyslipidemia or obesity).

\section{Method}

\section{Study design}

From February 2011 to October 2013, after discharge from coronary care units (usually from 3 to 10 days after the acute event), ACS patients at their first coronary event were recruited from three large Italian public hospitals (Azienda OspedalieroUniversitaria Careggi of Florence, Istituti Clinici di Perfezionamento Hospital of Milan, and Azienda Ospedaliera Bolognini of Seriate). Given that comparing CR and non-CR patients was not an objective of this research, the study population was composed of patients attending a CR program for greater ease of recruitment. Eligible patients were between age 30 and 80 and had sufficient Italian language skills. The exclusion criteria were: comorbidity with other major pathologies such as cancer, or the presence of cognitive deficits due to a diagnosed neurological disease. Those who fulfilled the 
eligibility criteria were recruited by physicians during their rehabilitation treatment at the hospital. After the first data collection, patients were re-evaluated at four subsequent follow-ups (after 6 months, 1, 2, and 3 years). At each time-point, anthropometric, clinical, and behavioral data were gathered: information on lifestyle before the onset of ACS was retrospectively collected during the first study visit, while that on adherence to pharmacological treatments was collected during the follow-ups.

The study was approved by the Bio-Ethics Committee of all the institutions involved in the research project. Each participant provided written informed consent prior to their enrollment.

\section{Measures}

Adherence to pharmacological treatments Participants' adherence to pharmacological treatments was examined by the Morisky Green Levine Medication Adherence Scale (MGLS; Morisky et al. 1986), a 4-item dichotomous (yes/no) measure of adherence to medications. The total scores on the MGLS range from 0 to 4 : a score of 0 indicates a low risk of nonadherence, scores of 1 or above reflect a medium risk of nonadherence to the pharmacological treatments, while scores of 3 or above indicate a high risk (Morisky et al. 1986). The MGLS evidenced fair psychometric properties (Morisky et al. 1986).

Diet Dietary behavior was measured using the Italian version of the Mediterranean Diet Scale (MDS; Steca et al. 2015; Trichopoulou et al. 2003). The MDS is a 9-item self-report questionnaire that measures the weekly consumption of nine foods using a 6-point Likert scale (from $1=$ Never to $6=$ More than three times per day). The consumption of both beneficial (i.e., vegetables, fruits, whole grains, fish, legumes, olive oil) and detrimental foods (i.e., more than two glasses of wine per day for men and more than one glass of wine for women, butter and margarine or vegetable oil other than olive oil, red or processed meat) was assessed. The sum of the recoded responses yielded the MDS, with higher scores indicating a healthier diet. A score of four and above shows good adherence to the Mediterranean diet and has been related to good health outcomes (Trichopoulou et al. 1995). The MDS has demonstrated good internal consistencies and construct validity (Steca et al. 2015; Trichopoulou et al. 2003).

Physical activity Physical activity was examined by the Italian version of the Rapid Assessment of Physical Activity Questionnaire (RAPA-Q; Topolski et al. 2006), a 7-item measure of frequency and intensity of participants' physical activity. The questionnaire uses a yes/no scale. The total score ranges from one (i.e., sedentary) to seven (i.e., regular and vigorous activity), with higher scores indicating a healthier amount of physical activity. Scores of 6 or 7 (i.e., at least
30 min of moderate to vigorous aerobic exercise five times a week) indicate the target amount of physical activity for cardiovascular prevention. Previous research has shown that RAPA-Q is a psychometrically sound questionnaire (Topolski et al. 2006).

Alcohol consumption behavior Participants' total alcohol intake was computed as the mean consumption of beer, wine (for both questions, the scale ratings were $0=$ "I do not drink wine/beer", 1 = "up to 2 glasses per day", 2 = "3 to 4 glasses per day", 3 = "more than 4 glasses per day"), and spirits (the scale ratings were $0=$ "I do not drink liquor", 1 = "a shot glass a few times per year", 2 = "a shot glass regularly (e.g., every week, after all meals)", 3 = "more than one shot glass regularly (e.g., every week, after all meals)"). Higher total scores reflect higher alcohol consumption.

Cigarette smoking behavior Participants' smoking behavior was measured with the question "How many cigarettes do you smoke per day?". The scale ratings were $0=$ "No cigarettes", 1 = "10 cigarettes or fewer per day", $2=$ " $11-20$ cigarettes per day", $3=$ "21-30 cigarettes per day", and $4=$ "31 or more cigarettes per day".

Anthropometric and clinical data Anthropometric and clinical parameters were measured according to European guidelines (e.g., Piepoli et al. 2016) and evaluated by routine standard hospital methods and procedures.

\section{Data analysis}

Changes in total MDS score (diet), levels of physical activity, and alcohol consumption from 6 months post-event to the 3year follow-up were evaluated using two-level hierarchical linear models (HLMs), while smoking status and adherence to pharmacological treatments (two dichotomous variables) were analyzed using hierarchical generalized linear models (HGLMs). H(G)LMs are one of the best statistical methods to examine longitudinal changes in nested data (Raudenbush and Bryk 2002). Their main advantage is the flexibility in handling missing data (Gallop and Tasca 2009), a common occurrence in longitudinal studies.

First, we ran a pattern mixture model to evaluate if data were missing at random and if having any missing data was significantly related to outcomes (Gallop and Tasca 2009). Then, we tested the hypotheses on lifestyle behaviors using piecewise regression models in $\mathrm{H}(\mathrm{G}) \mathrm{LM}$, in which two level1 "time" parameters were included to model the slope discontinuity from pre- to 6 months post-cardiovascular event (Singer and Willett 2003). Finally, data on adherence (from the 6-month to the 3-year follow-up) were analyzed using $\mathrm{H}(\mathrm{G}) \mathrm{LM}$, adding a linear time parameter and controlling for its starting values at the 6-month. 
H(G)LM analyses were adjusted for several confounding demographic and clinical variables, namely age, sex, working status (not working vs working), educational level (less than high school vs high school or higher), living status (alone vs with someone), marital status (single/widowed/divorced vs married), family history, hypertension, diabetes, dyslipidemia, and obesity (all the latter were: not present vs present). We additionally assessed and reported pseudo- $\mathrm{R}^{2}$, a measure of HLMs' effect sizes indicating the proportion of within-person variance accounted for by adding the linear parameters (Raudenbush and Bryk 2002). All multilevel models and further information on piecewise regressions are reported in the Appendix.

We controlled for the inflation of type I error using the Benjamini-Hochberg Procedure for False Discovery Rate (Benjamini and Hochberg 2000) on all the resulting $p$ values. Analyses were performed using the Statistical Package for Social Sciences (SPSS; IBM Corp., Boston, 2015) version 25.0 and Hierarchical Linear Models (HLM; SSI Inc., Lincolnwood, 2015) version 7.0.3. All statistical tests were two-tailed, and a $p \leq .05$ was considered statistically significant.

\section{Results}

\section{Study population}

A total of 300 consecutive patients with ACS admitted to a CR program and who fulfilled the eligibility criteria were invited to participate in the study, 25 of whom refused. Thus, the study population included 275 patients at their first coronary event, aged $57.1 \pm 7.87$ years; $84 \%$ were males and $16 \%$ females; their mean BMI was $27.2 \pm 4.1 \mathrm{~kg} / \mathrm{m}^{2}$, and their mean waist circumference [WC] was $96.5 \pm 11.1 \mathrm{~cm}$. All of them were Caucasian.

As regards drop-outs, $12.7 \%$ of patients were absent at the 6-month, $14.9 \%$ at the 1 -year, $20.7 \%$ at the 2 -year, and $33.1 \%$ at the 3-year follow-up (see Fig. 1 for the flow of participants through the study). In comparison, percentages of drop-outs at 1-year follow-up were similar to those reported in other European studies on ACS patients (e.g., Munyombwe et al. 2020). Causes of drop-out included: loss to follow-up, relocation, refusal and, in a small minority of cases, untraceability of the patient. It is worth noting that the statistical techniques adopted in this study enabled us to use all the data available and not only those provided by completers. Therefore, the final number of participants remained 275 . We further compared the sociodemographic and clinical characteristics of dropouts with those of completers through chi-square and Mann-Whitney U tests. After correcting for the inflation of $p$ values, we found no statistically significant differences between the two groups (see Tables 1 and 2 for means, SD, counts, proportions, and $p$ values). We additionally tested if the data was missing at random using a pattern mixture model (Gallop and Tasca 2009): the non-significant effects of the missing data pattern (dropouts vs completers) suggested that all data were missing at random and that the estimates of effects were unbiased by the presence of dropouts (Kristman et al. 2004). Thus, those who dropped out did not have worse lifestyle behaviors than completers.

Almost all patients were prescribed pharmacological treatment for ACS, consisting of antiplatelet drugs (99\% of patients), beta-blockers (89\%), statins $(97 \%)$, sartans or aceinhibitors (99\%). The pharmacological treatment did not significantly change over time (Steca et al. 2017). Finally, during the study period the percentage of patients re-hospitalized due to cardiac problems at each time point was lower than $6 \%$.

Further demographic and clinical variables are reported in Tables 1 and 2.

\section{Preliminary analyses}

Preliminary analyses found no univariate outliers or violations of normality in any of the continuous variables, except for "smoking status." Responses to this question were dichotomized (non-smoking/smoking) due to the non-normal distribution of cigarettes smoked per day. Further, only a small proportion of participants reported the highest risk of nonadherence (i.e., a total score $\geq 3$ on the MGLS; see Table 3); therefore, analyses were run considering total scores $\geq 1$ on the MGLS (which indexes individuals with a medium-to-high risk of non-adherence) as an occurrence.

Means and standard deviations for all variables across all time points are reported in Table 3.

\section{Longitudinal changes in adherence to pharmacological treatments}

Results highlighted longitudinal increases in non-adherence from 6 months post-treatment to the 3-year follow-up $(\mathrm{OR}=$ 1.26). Interestingly, marital status had a significant effect on the longitudinal slope of the dependent variable $(\beta=0.52 ; p=$ 0.005 , OR $=1.68$, suggesting that those who were not married worsened over time (i.e., experienced a progressively low adherence to pharmacological treatments), compared to those who were married. No other predictors were significant.

\section{Longitudinal changes in lifestyle behaviors}

As regards dietary behaviors, we found significant increases across all patients from pre-event to 6 months after (i.e., the "Time.1" slope $\beta_{10}$ ), and these increases were higher among those with hypertension $(\beta=0.85 ; p=0.005)$. However, those who were older had reduced increases $(\beta=-0.07 ; p=0.007)$, compared to those who were younger. We additionally found significant longitudinal decreases in the levels of dietary 
Fig. 1 Flow of participants through each stage of the study

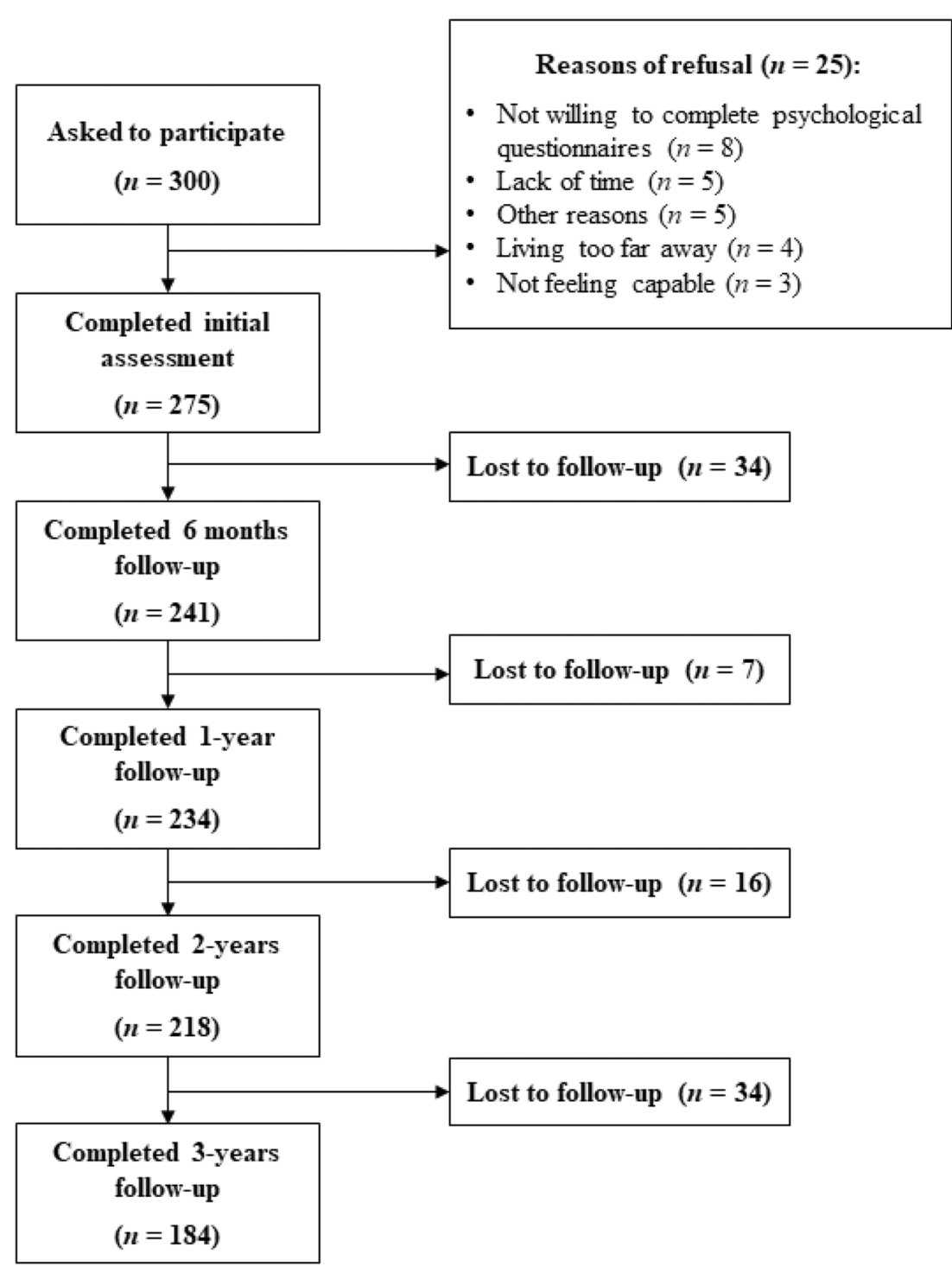

behaviors from the 6-month to the 3-year follow-up (i.e., the "Time.2" slope $\left.\beta_{20}\right)$; this decrease was smaller among older patients $(\beta=0.01 ; p=0.009)$ than younger ones and among those with hypertension $(\beta=-0.16 ; p=0.008)$. No other predictors were significant.

As regards physical activity, we found significant increases across all patients from pre-event to 6 months after; patients with higher educational levels $(\beta=1.42 ; p=0.004)$ had steeper increases compared to those with lower education. However, there was a significant longitudinal decrease in levels of physical activity from the 6-month to the 3-year follow-up. No other predictors were significant.

As regards alcohol consumption, before the onset of the cardiovascular disease younger individuals $(\beta=-0.02 ; p=$ $0.002)$, males $(\beta=-0.72 ; p<0.001)$ and workers $(\beta=0.38$; $p<0.001$ ) had worse alcohol outcomes. We found significant decreases in alcohol consumption across all patients from preevent to 6 months after. No longitudinal changes from the 6- month to the 3-year follow-up were found in this specific behavior. However, those with a diagnosis of dyslipidemia reported longitudinal increases over time in alcohol consumption $(\beta=0.07 ; p=0.004)$. No other predictors were significant.

As regards smoking behavior, before the onset of the cardiovascular disease, younger individuals $(\beta=-0.09$; $p<0.001$, Odds Ratio $[\mathrm{OR}]=0.92)$ and those who were mar$\operatorname{ried}(\beta=-1.51 ; p<0.001, \mathrm{OR}=0.23)$ smoked less. We found significant decreases in smoking behavior across all patients from pre-event to 6 months after $(\mathrm{OR}=<0.01)$; those who were married $(\beta=3.39 ; p=0.004, \mathrm{OR}=0.13)$ changed less, from pre-event to 6-months after. No longitudinal changes in this specific behavior were found from the 6-month to the 3 year follow-up. No further predictors were significant.

All regression coefficients, standard errors, $t$ and $p$ values for $\beta_{10}$ (i.e., the unstandardized regression coefficient for the average rate of growth from pre-event to 6 months after) and $\beta_{20}$ (i.e., the unstandardized regression coefficient for the 
Table 1 Sociodemographic characteristics of the entire sample $(N=275)$, completers $(N=184)$, and dropouts $(N=91)$

\begin{tabular}{lccc}
\hline Sociodemographic variables & ACS patients $(N=275)$ & Completers $(N=184)$ & Dropouts $(N=91)$ \\
\hline Age, mean (SD) & $57.1(7.87)$ & $57.1(8.03)$ & $56.9(7.60)$ \\
Gender, $n$ (\%) & $231(84)$ & $156(84.8)$ & $75(82.4)$ \\
$\quad$ Male & $44(16)$ & $28(15.2)$ & $16(17.6)$ \\
$\quad$ Female & $111(40.4)$ & $74(40.2)$ & $37(41.1)$ \\
$\quad$ Working status, $n(\%)$ & $163(59.3)$ & $110(59.8)$ & $53(58.9)$ \\
$\quad$ Working & $141(51.3)$ & $85(46.2)$ & $56(61.5)$ \\
$\quad$ Not working & $134(48.7)$ & $99(53.8)$ & $35(38.5)$ \\
Educational level, $n(\%)$ & $34(12.4)$ & $21(11.4)$ & $13(14.3)$ \\
$\quad$ Less than high school & $241(87.6)$ & $163(88.6)$ & $78(85.7)$ \\
$\quad$ High school or higher & $78(28.4)$ & $53(28.8)$ & $.017 *$ \\
Living status, $n$ (\%) & $197(71.6)$ & $131(71.2)$ & .89 \\
$\quad$ Alone & With someone & & $66(27.5)$ \\
Marital status, $n$ (\%) & Single/widowed/divorced & & .50 \\
$\quad$ Married & & & .82 \\
\hline
\end{tabular}

*This $p$ value was not significant after the Benjamini-Hochberg correction for false discovery rate

average rate of growth from the 6-month to the 3-year followup) slope parameters are reported in Table 4.

\section{Discussion}

Investigating longitudinal trajectories of lifestyle behaviors and adherence to pharmacological treatments among patients with cardiovascular diseases after their first acute coronary event represents one of the greatest challenges in preventive cardiology. In the current prospective cohort study, we found that - on average - the 275 newly diagnosed ACS patients experienced significant increases in the levels of healthy behaviors from before the cardiovascular event to 6 months after. However, from post-event to the 3-year follow-up, patients showed a significant decline in the levels of healthy dietary habits and physical activity, while no changes in alcohol consumption or smoking status were observed. In addition, adherence to pharmacological treatments showed a statistically significant decrease over time. Thus, years after their admission to CR programs, patients experience difficulties in adhering to their pharmacological treatments and to some of the most challenging recommended lifestyle modifications, namely healthy dietary habits and physical activity.

Our results are in agreement with and greatly extend results from previous studies (e.g., Crowley et al. 2015; Kotseva et al. 2019; Molloy et al. 2014; Peersen et al. 2017; Urbinati et al. 2014; Wójcicki et al. 2020). For example, recent reports suggest that $40-50 \%$ of patients with established cardiovascular diseases do not consistently take their recommended medications, suggesting that non-adherence is a common occurrence among these individuals (Crowley et al. 2015; Molloy et al. 2014). Wójcicki et al. (2020) recently showed that patients

Table 2 Clinical characteristics of the sample $(N=275)$, completers $(N=184)$, and dropouts $(N=91)$

\begin{tabular}{|c|c|c|c|c|}
\hline Clinical variables & ACS patients $(N=275)$ & Completers $(N=184)$ & Dropouts $(N=91)$ & $p$ value \\
\hline BMI, mean (SD) & $27.2(4.1)$ & $26.8(4.0)$ & $28.0(4.2)$ & $.013 *$ \\
\hline Waistline, mean (SD) & $96.5(11.1)$ & $95.2(11.0)$ & $99.2(10.8)$ & $.005 *$ \\
\hline Clinical presentation, $n(\%)$ & & & & .88 \\
\hline Non-ST elevation myocardial infarction (NSTEMI) & $54(19.8)$ & $37(20.2)$ & $17(18.9)$ & \\
\hline ST elevation myocardial infarction (STEMI) & $196(71.8)$ & $131(71.6)$ & $65(72.2)$ & \\
\hline Unstable angina & $23(8.5)$ & $15(8.2)$ & $8(8.9)$ & \\
\hline Percutaneous coronary intervention, $n(\%)$ & $258(94.5)$ & $176(95.7)$ & $82(92.1)$ & .36 \\
\hline Patients with at least one stent, $n(\%)$ & $263(96)$ & $177(96.2)$ & $86(94.5)$ & .46 \\
\hline \multicolumn{5}{|l|}{ Risk factors, $n(\%)$} \\
\hline Hypertension & $127(46.5)$ & $80(43.5)$ & $47(52.8)$ & .15 \\
\hline Dyslipidemia & $143(52.4)$ & $92(50)$ & $51(57.3)$ & .26 \\
\hline Diabetes & $47(17.2)$ & $29(15.8)$ & $18(20.2)$ & .36 \\
\hline Obesity & $43(15.8)$ & $20(46.5)$ & $23(53.5)$ & $.034 *$ \\
\hline Family history & $108(39.3)$ & $73(39.7)$ & $35(39.3)$ & .96 \\
\hline Systolic blood pressure (SBP), mean (SD) & $115.9(13.9)$ & $116.2(13.2)$ & $115.8(14.3)$ & .61 \\
\hline Diastolic blood pressure (DBP), mean (SD) & $72.9(8.5)$ & $72.2(8.7)$ & $73.3(8.4)$ & .39 \\
\hline
\end{tabular}

*These $p$-values were not significant after the Benjamini-Hochberg correction for false discovery rate 
Table 3 Means, standard deviation, and frequencies for the behavioral outcomes (diet, physical activity, alcohol consumption behavior, cigarette smoking behavior, and adherence to pharmacological treatments) during all time points (pre-event, 6-month, 1-, 2-, and 3-year follow-up)

\begin{tabular}{|c|c|c|c|c|c|c|c|c|c|c|}
\hline & \multicolumn{2}{|c|}{ Pre * } & \multicolumn{2}{|c|}{6 months } & \multicolumn{2}{|c|}{1 year } & \multicolumn{2}{|c|}{2 years } & \multicolumn{2}{|c|}{3 years } \\
\hline & $N$ & Mean (SD) & $N$ & Mean (SD) & $N$ & Mean (SD) & $N$ & Mean (SD) & $N$ & Mean (SD) \\
\hline Diet & 275 & $3.40(1.40)$ & 240 & $4.37(1.47)$ & 234 & $4.30(1.47)$ & 218 & $4.13(1.62)$ & 184 & $4.09(1.58)$ \\
\hline Physical activity & 275 & $4.20(2.02)$ & 240 & $5.47(1.79)$ & 234 & $5.36(1.80)$ & 217 & $5.17(1.91)$ & 183 & $5.12(1.93)$ \\
\hline Alcohol consumption & 275 & $1.68(0.79)$ & 240 & $1.33(0.71)$ & 231 & $1.33(0.73)$ & 213 & $1.24(0.73)$ & 181 & $1.26(0.73)$ \\
\hline Smoking behavior: $n(\%)$ & 274 & $154(56.2 \%)$ & 238 & $31(13.0 \%)$ & 230 & $28(12.2 \%)$ & 213 & $28(13.1 \%)$ & 180 & $21(11.7 \%)$ \\
\hline MGLS $\geq 3: n(\%)$ & 1 & 1 & 238 & $0(0 \%)$ & 231 & $1(0.4 \%)$ & 217 & $2(0.9 \%)$ & 182 & $6(3.3 \%)$ \\
\hline MGLS $\geq 1: n(\%)$ & 1 & 1 & 238 & $91(38.2 \%)$ & 231 & $89(38.5 \%)$ & 217 & $92(42.5 \%)$ & 182 & $77(42.3 \%)$ \\
\hline
\end{tabular}

*Data on lifestyle behaviors at pre-event were collected retrospectively once patients were hospitalized. Smoking behavior refers to the number of smokers. MGLS, Morisky Green Levine Medication Adherence Scale; A MGLS score of 1 or above is indicative of a medium risk of non-adherence to pharmacological treatments, while a score of 3 or above is indicative of a high risk

with a cardiovascular disease experience insufficient changes in individual lifestyle after a percutaneous coronary intervention, and report an overall poor awareness of the cardiovascular risk factors. Moreover, a large European cross-sectional study on 8261 patients interviewed approximately 1 year after their first cardiac event showed that most of them failed to achieve the healthy lifestyle targets set by the Joint European Societies of Cardiology (Kotseva et al. 2019).

In our study, adherence to pharmacological treatments decreased over time, suggesting that the risk of (moderate) nonadherence significantly increases up to 3 years post-follow-up. Moreover, being married was a significant protective factor: those who were married reported an increase in adherence over time in comparison to non-married participants, who experienced a worsening in this specific outcome. This result confirms the crucial protective role of social support in fostering a high adherence to treatment in cardiovascular pathologies as suggested by previous studies (Magrin et al. 2014). It is worth noting that the number of those considered at the highest risk of non-adherence (i.e., with scores $\geq 3$ on the Medication Adherence Scale) was very low, with few occurrences during the latter time points (see Table 3 ). On the contrary, a percentage of patients ranging from $38 \%$ to $42 \%$ self-reported a moderate risk of non-adherence to pharmacological treatments (i.e., with scores $\geq 1$ on the Medication Adherence Scale), suggesting that at least $1 / 3$ of patients with ACS sometimes forget to take medication, or stop taking it due to its adverse effects.

As regards dietary habits, mean levels of the Mediterranean Diet Scale scores after the cardiovascular event were all above the suggested cut-off score of four (see Table 3), which has been related to positive health outcomes (Trichopoulou et al. 1995). However, this adherence declined over time. We found that being older was associated with greater difficulties in changing dietary habits from pre- to 6 months post-event. Despite this, older adults experienced less change in this habit up to 3-years follow-up, compared to younger adults: in other words, they were more perseverant and complied better to this specific regimen, once a positive change was reached. We suppose this is due to a greater tendency to adopt stable behaviors in older adults as well as to a deeper awareness of the disease, which in turn could be underestimated by younger adults. Furthermore, we may suppose that they have also received more recommendations in view of their condition of greater risk due to age. Interestingly, those with hypertension showed the opposite pattern of change (i.e., an initial steeper increase in this healthy behavior followed by a worsening up to the 3-year follow-up). Given that hypertension is a major risk factor for cardiovascular disease (Middeke et al. 2008), one could argue that these patients received more healthrelated suggestions at intake, which initially led to better outcomes that were later disregarded as time went on. We need to remember that hypertension is often defined as a silent pathology as hypertensive people usually do not experience serious symptoms (Middeke et al. 2008). Therefore, this condition may contribute to patients' underestimation of the importance of preventive behaviors.

Adherence to good levels of physical activity is one of the most difficult goals to reach and maintain among patients with CVDs (Kotseva et al. 2019; Tang et al. 2013), with only 17\% of the individuals affected by a cardiovascular disease meeting the guideline-recommended amounts per week (Tang et al. 2013). In our study, patients reported sub-optimal amounts of physical activity at each time-point (i.e., with a total RAPA-Q score below 6 or 7 points; see Table 3); moreover, the gains reached soon after the CR program declined linearly over the course of 3 years. Higher educational levels predicted higher physical activity from pre- to 6 months post-event, confirming the protective effect of this specific demographic variable (Aaby et al. 2017; Cutler and Lleras-Muney 2010; Veronesi et al. 2016).

Findings suggest that smoking status and alcohol consumption are lifestyle behaviors whose change seems easier to maintain. We found that after the acute cardiovascular event, there were no significant changes in their levels up to the three-year follow-up. Our results are partly in accordance with a previous cross-sectional study performed in the US, where more than $70 \%$ of persons with known coronary heart disease interviewed approximately 1 year after the event were non-smokers and at the recommended levels of alcohol consumption (Tang et al. 2013). We additionally found few predictors of change in both 
Table 4 Fixed effects for the longitudinal changes in behavioral outcomes (diet, physical activity, alcohol consumption behavior, cigarette smoking behavior, and adherence to pharmacological treatments)

\begin{tabular}{|c|c|c|c|c|c|c|c|c|c|c|}
\hline \multirow[t]{2}{*}{ Variable } & \multicolumn{10}{|c|}{ Fixed effects } \\
\hline & $\beta_{10}$ & $S E$ & $t$ values & $p$ & $\beta_{20}$ & $S E$ & $t$ values & $p$ & $d f$ & $R^{2}$ \\
\hline Diet & 1.62 & 0.15 & 11.05 & $<0.001$ & -0.11 & 0.028 & -3.90 & $<0.001$ & 258 & 0.25 \\
\hline Physical activity & 2.13 & 0.23 & 9.24 & $<0.001$ & -0.12 & 0.04 & -3.087 & 0.002 & 258 & 0.43 \\
\hline Alcohol consumption & -0.63 & 0.07 & -8.84 & $<0.001$ & -0.013 & 0.012 & -1.13 & 0.26 & 258 & 0.31 \\
\hline Smoking behavior & -6.10 & 0.46 & -13.27 & $<0.001$ & -0.09 & 0.12 & -0.78 & 0.43 & 258 & 1 \\
\hline Adherence & 0.23 & 0.07 & 3.55 & $<0.001$ & $\backslash$ & 1 & 1 & 1 & 220 & 1 \\
\hline
\end{tabular}

$\beta_{10}=$ unstandardized regression coefficient for the average rate of growth from pre-event to 6 months after. $\beta_{20}=$ unstandardized regression coefficient for the average rate of growth from the 6-month up to the 3-year follow-up. SE, standard error of the regression coefficient; $d f$, degrees of freedom $(d f$ are less than expected due to a few missing data at level 2). $\mathrm{R}^{2}$ refers to pseudo- $\mathrm{R}^{2}$ indicating the proportion of within-person variance accounted for by adding the "Time.1" and "Time.2" parameter to the model. Adherence was measured through the Morisky Green Levine Medication Adherence Scale

behaviors: for example, those with a diagnosis of dyslipidemia reported longitudinal increases over time in alcohol consumption. In addition, those who were married were more likely to continue smoking from pre- to 6 months post-event, because their odds of being smokers were already lower at intake (i.e., before the cardiovascular event) compared to those who were not married.

The current study has a series of limitations that must be considered in interpreting our results. First, unhealthy lifestyles before the onset of cardiovascular disease were measured retrospectively. This approach may limit the reliability of our data, for example due to the patients' over- or underestimation of their real healthy lifestyles prior to the onset of the cardiovascular disease. Second, diet, physical activity, alcohol consumption, and cigarette smoking were examined through self-report questions and measures and not through an objective behavioral assessment. The medical and psychological literature, however, has widely adopted a self-report measurement of these healthy behaviors (Steca et al. 2015, 2017; Topolski et al. 2006; Trichopoulou et al. 2003), and any other methodology would be hard to be maintained in an extended longitudinal study such as the current one. Third, our study population was composed exclusively of Italian patients, the vast majority of whom were male (84\%), partly limiting the generalizability of the current findings to other ethnicities and to the female population. However, our sample is representative of the gender distribution of ACS in Europe (e.g., Munyombwe et al. 2020).

Despite these limitations, our study has a number of strengths and reports several original findings that suggest important implications for further research and practical applications in behavioral intervention for ACS patients. First, the study involved a homogeneous population of patients at their first cardiovascular event. This is a crucial moment not only in their lives, as the event is dramatic and unexpected most of the time, but also in terms of cardiovascular secondary prevention. On one hand, the homogeneity of the sample may have limited the generalizability of the findings, but on the other hand, it guarantees that results completely apply only to ACS patients at their first cardiovascular event. Indeed, our conclusions are difficult to draw from those studies that involve large groups of patients with very different cardiovascular diseases and clinical characteristics. Second, the study considers a multiplicity of health-related behaviors, unlike other research that focused exclusively on a specific behavior. This is important to detect common pathways and associations. Third, patients' lifestyles were analyzed over a long period of time ( 3 years), enabling us to investigate how health-related behaviors evolve over time. Our results clearly show that the greatest difficulty faced by patients is not changing lifestyles following a coronary event but maintaining the improvement over time, probably due to the additional efforts required to preserve these specific behaviors and healthy lifestyles. Furthermore, they suggest that this maintenance is more difficult for individuals with specific sociodemographic and/or clinical characteristics than others. In conclusion, we suggest to promptly and accurately evaluate patients' lifestyles upon admission to the hospital, in order to identify possible unhealthy behaviors and their co-occurrence. Subsequently, we suggest delivering appropriate multitarget psychological intervention programs that have to be repeated over time, especially after the first year subsequent to the cardiovascular event. Those programs should aim at fostering patients' capabilities to self-regulate their behaviors and increase their beliefs about their self-efficacy (Greco et al. 2014; Vellone et al. 2016), supposedly improving the prognosis of patients by reducing mortality and the risk of recurrent events over time.

Funding Information Open access funding provided by Università degli Studi di Bergamo within the CRUI-CARE Agreement. This study was supported by a FIRB ("Futuro in Ricerca") Grant from the Italian Ministry of Instruction, University and Research (ref. RBFR08YVUL). The funders had no role in the study's design, data collection and analysis, decision to publish, or preparation of the manuscript.

\section{Compliance with ethical standards}

Ethics approval All procedures performed in studies involving human participants were in accordance with the ethical standards of the institutional and/or national research committee and with the 1964 Helsinki declaration and its later amendments or comparable ethical standards. 
Consent to participate Informed consent was obtained from all individual participants included in the study.

Conflict of interest The authors declare that they have no conflict of interest.

\section{Appendix}

We assessed the uncontrolled effects of the pre- to 6 months post-event changes in lifestyle behaviors (Diet, Physical activity, Alcohol consumption, and Smoking status) using piecewise regression models with two "time" parameters at level 1.
The first "time" parameter (Time.1) was set at " 0 " for pre-event and " 0.6 " representing 6 months after the event (i.e., the second evaluation), and " 0.6 " also for all subsequent measurement occasions. This modeled a linear increase from pre- to 6 months post-event but no further changes in behavioral data.

The second time parameter (Time.2) was set at " 0 " for pre- and 6 months post-event, while the other time points were then coded as "1.2,"“2.4," and "3.6" to represent the 1-year, 2-year, and 3-year follow-up. This modeled a linear increase from 6 months post-event to the 3-year follow-up.

\section{HLM 2-level models for lifestyle behaviors}

\section{Level-1 Model}

$\mathrm{Y}_{\mathrm{ti}}=\pi_{0 \mathrm{i}}+\pi_{1 \mathrm{i}} *\left(\right.$ TIME. $\left.1_{\mathrm{ti}}\right)+\pi_{2 \mathrm{i}} *\left(\right.$ TIME. $\left.2_{\mathrm{ti}}\right)+\mathrm{e}_{\mathrm{ti}}$

\section{Level-2 Model}

$$
\begin{aligned}
& \left.\pi_{0 \mathrm{i}}=\beta_{00}+\beta_{01} *\left(\mathrm{AGE}_{\mathrm{i}}\right)+\beta_{02} *\left(\mathrm{SEX}_{\mathrm{i}}\right)+\beta_{03} *\left(\mathrm{WORKING}_{-} \mathrm{STATUS}\right)_{\mathrm{i}}\right)+\beta_{04} * \\
& \left(\text { MARITAL_STATUS }_{\mathrm{i}}\right)+\beta_{05} *\left(\text { EDUCATION }_{\mathrm{i}}\right)+\beta_{06} *\left(\text { LIVING_STATUS }_{\mathrm{i}}\right)+\beta_{07} * \\
& \left(\text { FAMILY_HISTORY }_{\mathrm{i}}\right)+\beta_{08} *\left(\text { HYPERTENSION }_{\mathrm{i}}\right)+\beta_{09} *\left(\text { DIABETES }_{\mathrm{i}}\right)+\beta_{010} * \\
& \left(\text { DYSLIPIDEMIA }_{\mathrm{i}}\right)+\beta_{011} *\left(\text { OBESITY }_{\mathrm{i}}\right)+\mathrm{r}_{0 \mathrm{i}} \\
& \pi_{1 \mathrm{i}}=\beta_{10}+\beta_{11} *\left(\mathrm{AGE}_{\mathrm{i}}\right)+\beta_{12} *\left(\mathrm{SEX}_{\mathrm{i}}\right)+\beta_{13} *\left(\mathrm{WORKING}_{-} \mathrm{STATUS}_{\mathrm{i}}\right)+\beta_{14} * \\
& \left(\text { MARITAL_STATUS }_{\mathrm{i}}\right)+\beta_{15} *\left(\text { EDUCATION }_{\mathrm{i}}\right)+\beta_{16} *\left(\text { LIVING_STATUS }_{\mathrm{i}}\right)+\beta_{17} * \\
& \left(\text { FAMILY_HISTORY }_{\mathrm{i}}\right)+\beta_{18} *\left(\text { HYPERTENSION }_{\mathrm{i}}\right)+\beta_{19} *\left(\text { DIABETES }_{\mathrm{i}}\right)+\beta_{110} * \\
& \left(\text { DYSLIPIDEMIA }_{\mathrm{i}}\right)+\beta_{111} *\left(\text { OBESITY }_{\mathrm{i}}\right)+\mathrm{r}_{1 \mathrm{i}} \\
& \pi_{2 \mathrm{i}}=\beta_{20}+\beta_{21} *\left(\mathrm{AGE}_{\mathrm{i}}\right)+\beta_{22} *\left(\mathrm{SEX}_{\mathrm{i}}\right)+\beta_{23} *\left(\text { WORKING_STATUS }_{\mathrm{i}}\right)+\beta_{24} * \\
& \left(\text { MARITAL_STATUS }_{\mathrm{i}}\right)+\beta_{25} *\left(\text { EDUCATION }_{\mathrm{i}}\right)+\beta_{26} *\left(\text { LIVING_STATUS }_{\mathrm{i}}\right)+\beta_{27} * \\
& \left(\text { FAMILY_HISTORY }_{\mathrm{i}}\right)+\beta_{28} *\left(\text { HYPERTENSION }_{\mathrm{i}}\right)+\beta_{29} *\left(\text { DIABETES }_{\mathrm{i}}\right)+\beta_{210} * \\
& \left(\text { DYSLIPIDEMIA }_{\mathrm{i}}\right)+\beta_{211} *\left(\text { OBESITY }_{\mathrm{i}}\right)+\mathrm{r}_{2 \mathrm{i}}
\end{aligned}
$$




\section{HGLM 2-level model for Smoking status}

\section{Level-1 Model}

$$
\begin{aligned}
& \operatorname{Prob}\left(\mathrm{Y}_{t i}=1 \mid \pi_{i}\right)=\phi_{t i} \\
& \log \left[\phi_{t i} /\left(1-\phi_{t i}\right)\right]=\eta_{t i} \\
& \eta_{t i}=\pi_{0 i}+\pi_{l i} *\left(\text { TIME. } 1_{t i}\right)+\pi_{2 i} *\left(\text { TIME. } 2_{t i}\right)
\end{aligned}
$$

\section{Level-2 Model}

$$
\begin{aligned}
\pi_{0 \mathrm{i}}= & \beta_{00}+\beta_{01} *\left(\mathrm{AGE}_{\mathrm{i}}\right)+\beta_{02} *\left(\mathrm{SEX}_{\mathrm{i}}\right)+\beta_{03} *\left(\mathrm{WORKING}_{-} \mathrm{STATUS}_{\mathrm{i}}\right)+\beta_{04} * \\
& \left(\text { MARITAL_STATUS }_{\mathrm{i}}\right)+\beta_{05} *\left(\text { EDUCATION }_{\mathrm{i}}\right)+\beta_{06} *\left(\text { LIVING_STATUS }_{\mathrm{i}}\right)+\beta_{07} * \\
& \left(\text { FAMILY_HISTORY }_{\mathrm{i}}\right)+\beta_{08} *\left(\text { HYPERTENSION }_{\mathrm{i}}\right)+\beta_{09} *\left(\text { DIABETES }_{\mathrm{i}}\right)+\beta_{010} * \\
& \left(\text { DYSLIPIDEMIA }_{\mathrm{i}}\right)+\beta_{011} *\left(\text { OBESITY }_{\mathrm{i}}\right)+\mathrm{r}_{0 \mathrm{i}}
\end{aligned}
$$

$$
\begin{aligned}
\pi_{1 \mathrm{i}}= & \beta_{10}+\beta_{11} *\left(\mathrm{AGE}_{\mathrm{i}}\right)+\beta_{12} *\left(\mathrm{SEX}_{\mathrm{i}}\right)+\beta_{13} *\left(\mathrm{WORKING}_{-} \mathrm{STATUS}_{\mathrm{i}}\right)+\beta_{14} * \\
& \left(\text { MARITAL_STATUS }_{\mathrm{i}}\right)+\beta_{15} *\left(\text { EDUCATION }_{\mathrm{i}}\right)+\beta_{16} *\left(\text { LIVING_STATUS }_{\mathrm{i}}\right)+\beta_{17} * \\
& \left(\text { FAMILY_HISTORY }_{\mathrm{i}}\right)+\beta_{18} *\left(\text { HYPERTENSION }_{\mathrm{i}}\right)+\beta_{19} *\left(\text { DIABETES }_{\mathrm{i}}\right)+\beta_{110} * \\
& \left(\text { DYSLIPIDEMIA }_{\mathrm{i}}\right)+\beta_{111} *\left(\text { OBESITY }_{\mathrm{i}}\right)+\mathrm{r}_{1 \mathrm{i}}
\end{aligned}
$$

$$
\begin{aligned}
\pi_{2 \mathrm{i}}= & \beta_{20}+\beta_{21} *\left(\mathrm{AGE}_{\mathrm{i}}\right)+\beta_{22} *\left(\mathrm{SEX}_{\mathrm{i}}\right)+\beta_{23} *\left(\text { WORKING_STATUS }_{\mathrm{i}}\right)+\beta_{24} * \\
& \left(\text { MARITAL_STATUS }_{\mathrm{i}}\right)+\beta_{25} *\left(\text { EDUCATION }_{\mathrm{i}}\right)+\beta_{26} *\left(\text { LIVING_STATUS }_{\mathrm{i}}\right)+\beta_{27} * \\
& \left(\text { FAMILY_HISTORY }_{\mathrm{i}}\right)+\beta_{28} *\left(\text { HYPERTENSION }_{\mathrm{i}}\right)+\beta_{29} *\left(\text { DIABETES }_{\mathrm{i}}\right)+\beta_{210} * \\
& \left(\text { DYSLIPIDEMIA }_{\mathrm{i}}\right)+\beta_{211} *\left(\text { OBESITY }_{\mathrm{i}}\right)+\mathrm{r}_{2 \mathrm{i}}
\end{aligned}
$$




\section{Level-1 Model}

$$
\begin{aligned}
& \operatorname{Prob}\left(\mathrm{Y}_{t i}=1 \mid \pi_{i}\right)=\phi_{t i} \\
& \log \left[\phi_{t i} /\left(1-\phi_{t i}\right)\right]=\eta_{t i} \\
& \eta_{t i}=\pi_{0 i}+\pi_{l i} *\left(T I M E_{t i}\right)
\end{aligned}
$$

\section{Level-2 Model}

$$
\begin{aligned}
& \pi_{0 \mathrm{i}}=\beta_{00}+\beta_{01} *\left(\mathrm{AGE}_{\mathrm{i}}\right)+\beta_{02} *\left(\mathrm{SEX}_{\mathrm{i}}\right)+\beta_{03} *\left(\mathrm{WORKING}_{-} \mathrm{STATUS}_{\mathrm{i}}\right)+\beta_{04} * \\
& \left(\text { MARITAL_STATUS }_{\mathrm{i}}\right)+\beta_{05} *\left(\text { EDUCATION }_{\mathrm{i}}\right)+\beta_{06} *\left(\text { LIVING_STATUS }_{\mathrm{i}}\right)+\beta_{07} * \\
& \left(\text { FAMILY_HISTORY }_{\mathrm{i}}\right)+\beta_{08} *\left(\text { ADHERENCE_T1 }_{\mathrm{i}}\right)+\beta_{09} *\left(\text { HYPERTENSION }_{\mathrm{i}}\right)+\beta_{010} * \\
& \left(\text { DIABETES }_{\mathrm{i}}\right)+\beta_{011} *\left(\text { DYSLIPIDEMIA }_{\mathrm{i}}\right)+\beta_{012} *\left(\text { OBESITY }_{\mathrm{i}}\right)+\mathrm{r}_{0 \mathrm{i}}
\end{aligned}
$$

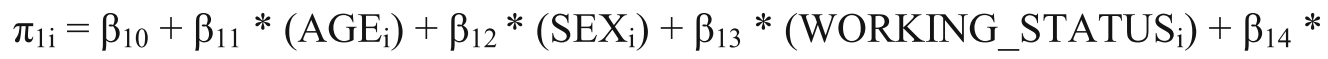

$$
\begin{aligned}
& \left(\text { MARITAL_STATUS }_{\mathrm{i}}\right)+\beta_{15} *\left(\text { EDUCATION }_{\mathrm{i}}\right)+\beta_{16} *\left(\text { LIVING_STATUS }_{\mathrm{i}}\right)+\beta_{17} * \\
& \left(\text { FAMILY_HISTORY }_{\mathrm{i}}\right)+\beta_{18} *\left(\text { ADHERENCE_T1 }_{\mathrm{i}}\right)+\beta_{19} *\left(\text { HYPERTENSION }_{\mathrm{i}}\right)+\beta_{110} * \\
& \left(\text { DIABETES }_{\mathrm{i}}\right)+\beta_{111} *\left(\text { DYSLIPIDEMIA }_{\mathrm{i}}\right)+\beta_{112} *\left(\text { OBESITY }_{\mathrm{i}}\right)+\mathrm{r}_{1 \mathrm{i}}
\end{aligned}
$$

Note: $\mathrm{Y}=$ Dependent variable (Dietary behavior, Physical activity, and Alcohol consumption behavior); All level-2 variables were centered around the grand mean.

Note. $\mathrm{Y}=$ Dependent variable (Cigarette smoking behavior, dichotomized); All level-2 variables were centered around the grand mean.

We assessed the controlled effects of the changes in adherence to pharmacological treatments from the 6-month to the 3year follow-up using an HLM model with a single "time" parameter at level $1(0=6$-months; $1=1$ year follow-up; $2=2$ years follow-up; $3=3$-years follow-up), controlling for adherence at the first time-point (i.e., baseline).

$\mathrm{Y}=$ Dependent variable (Adherence, dichotomized); All level-2 variables were centered around the grand mean.

Open Access This article is licensed under a Creative Commons Attribution 4.0 International License, which permits use, sharing, adaptation, distribution and reproduction in any medium or format, as long as you give appropriate credit to the original author(s) and the source, provide a link to the Creative Commons licence, and indicate if changes were made. The images or other third party material in this article are included in the article's Creative Commons licence, unless indicated otherwise in a credit line to the material. If material is not included in the article's Creative Commons licence and your intended use is not permitted by statutory regulation or exceeds the permitted use, you will need to obtain permission directly from the copyright holder. To view a copy of this licence, visit http://creativecommons.org/licenses/by/4.0/.

\section{References}

Aaby A, Friis K, Christensen B, Rowlands G, Maindal HT (2017) Health literacy is associated with health behaviour and self-reported health: a large population-based study in individuals with cardiovascular disease. Eur J Prev Cardiol 24:1880-1888. https://doi.org/10.1177/ 2047487317729538

Benjamini Y, Hochberg Y (2000) On the adaptive control of the false discovery rate in multiple testing with independent statistics. J Educ Behav Stat 25:60-83. https://doi.org/10.3102/10769986025001060

Chow CK, Jolly S, Rao-Melacini P, Fox KA, Anand SS, Yusuf S (2010) Association of diet, exercise, and smoking modification with risk of early 
cardiovascular events after acute coronary syndromes. Circulation 121: 750-758. https://doi.org/10.1161/circulationaha.109.891523

Crowley MJ, Zullig LL, Shah BR, Shaw RJ, Lindquist JH, Peterson ED, Bosworth HB (2015) Medication non-adherence after myocardial infarction: an exploration of modifying factors. J Gen Intern Med 30:83-90. https://doi.org/10.1007/s11606-014-3072-x

Cutler DM, Lleras-Muney A (2010) Understanding differences in health behaviors by education. J Health Econ 29:1-28. https://doi.org/10. 1016/j.jhealeco.2009.10.003

Erhardt L (2009) Cigarette smoking: an undertreated risk factor for cardiovascular disease. Atherosclerosis 205:23-32. https://doi.org/10. 1016/j.atherosclerosis.2009.01.007

ESC Scientific Document Group et al (2017) European Society of Cardiology: cardiovascular disease statistics 2017. Eur Heart J 39: 508-579. https://doi.org/10.1093/eurheartj/ehx628

Fattirolli $\mathrm{F}$ et al (2018) What constitutes the 'minimal care' interventions of the nurse, physiotherapist, dietician and psychologist in cardiovascular rehabilitation and secondary prevention: a position paper from the Italian Association for Cardiovascular Prevention, Rehabilitation and Epidemiology. Eur J Prev Cardiol 25:17991810. https://doi.org/10.1177/2047487318789497

Ford ES et al (2007) Explaining the decrease in U.S. deaths from coronary disease, 1980-2000. N Engl J Med 356:2388-2398. https://doi. org/10.1056/NEJMsa053935

Gallop R, Tasca GA (2009) Multilevel modeling of longitudinal data for psychotherapy researchers: II. The complexities. Psychother Res 19: 438-452. https://doi.org/10.1080/10503300902849475

Greco A et al (2014) Predicting depression from illness severity in cardiovascular disease patients: self-efficacy beliefs, illness perception, and perceived social support as mediators. Int J Behav Med 21:221229. https://doi.org/10.1007/s12529-013-9290-5

Ibanez B et al (2017) 2017 ESC guidelines for the management of acute myocardial infarction in patients presenting with ST-segment elevation: the task force for the management of acute myocardial infarction in patients presenting with ST-segment elevation of the European Society of Cardiology (ESC). Eur Heart J 39:119-177. https://doi.org/10.1093/eurheartj/ehx393

Kotseva $\mathrm{K}$ et al (2019) Lifestyle and impact on cardiovascular risk factor control in coronary patients across 27 countries: results from the European Society of Cardiology ESC-EORP EUROASPIRE V registry. Eur J Prev Cardiol 26:824-835. https://doi.org/10.1177/ 2047487318825350

Kristman V, Manno M, Cote P (2004) Loss to follow-up in cohort studies: how much is too much? Eur J Epidemiol 19:751-760. https:// doi.org/10.1023/b:ejep.0000036568.02655.f8

Lloyd-Jones Donald M et al (2010) Defining and setting national goals for cardiovascular health promotion and disease reduction. Circulation 121: 586-613. https://doi.org/10.1161/CIRCULATIONAHA.109.192703

Magrin ME et al (2014) Social support and adherence to treatment in hypertensive patients: a meta-analysis. Ann Behav Med 49:307318. https://doi.org/10.1007/s12160-014-9663-2

Middeke M, Lemmer B, Schaaf B, Eckes L (2008) Prevalence of hypertension-attributed symptoms in routine clinical practice: a general practitioners-based study. J Hum Hypertens 22:252-258. https://doi.org/10.1038/sj.jhh.1002305

Molloy GJ, Messerli-Bürgy N, Hutton G, Wikman A, Perkins-Porras L, Steptoe A (2014) Intentional and unintentional non-adherence to medications following an acute coronary syndrome: a longitudinal study. J Psychosom Res 76:430-432. https://doi.org/10.1016/j. jpsychores.2014.02.007

Morisky DE, Green LW, Levine DM (1986) Concurrent and predictive validity of a self-reported measure of medication adherence. Med Care 24:67-74

Munyombwe T et al (2020) Quality of life trajectories in survivors of acute myocardial infarction: a national longitudinal study. Heart 106:33-39. https://doi.org/10.1136/heartjnl-2019-315510
Nabel EG, Braunwald E (2012) A tale of coronary artery disease and myocardial infarction. N Engl J Med 366:54-63. https://doi.org/ 10.1056/NEJMra1112570

Peersen K et al (2017) The role of cardiac rehabilitation in secondary prevention after coronary events. Eur J Prev Cardiol 24:1360 1368. https://doi.org/10.1177/2047487317719355

Piepoli MF et al (2016) 2016 European guidelines on cardiovascular disease prevention in clinical practice: the sixth joint task force of the European Society of Cardiology and Other Societies on Cardiovascular Disease Prevention in Clinical Practice. Eur Heart J 37:2315-2381. https://doi.org/10.1093/eurheartj/ehw106

Raudenbush SW, Bryk AS (2002) Hierarchical linear models: applications and data analysis methods, 2nd edn. Sage, Thousand Oaks

Ruff CT, Braunwald E (2010) The evolving epidemiology of acute coronary syndromes. Nat Rev Cardiol 8:140. https://doi.org/10.1038/ nrcardio. 2010.199

Singer JD, Willett JB (2003) Applied longitudinal data analysis: modeling change and event occurrence. Oxford University Press, London

Steca P et al (2015) Changes in dietary behavior among coronary and hypertensive patients: a longitudinal investigation using the health action process approach. Appl Psychol Health Well Being 7:316339. https://doi.org/10.1111/aphw.12050

Steca $\mathrm{P}$ et al (2017) Stability and change of lifestyle profiles in cardiovascular patients after their first acute coronary event. PLoS One 12: e0183905. https://doi.org/10.1371/journal.pone.0183905

Tang L, Patao C, Chuang J, Wong ND (2013) Cardiovascular risk factor control and adherence to recommended lifestyle and medical therapies in persons with coronary heart disease (from the National Health and Nutrition Examination Survey 2007-2010). Am J Cardiol 112:11261132. https://doi.org/10.1016/j.amjcard.2013.05.064

Tea V et al (2018) Appropriate secondary prevention and clinical outcomes after acute myocardial infarction according to atherothrombotic risk stratification: the FAST-MI 2010 registry. Eur J Prev Cardiol 26:411419. https://doi.org/10.1177/2047487318808638

Topolski TD, LoGerfo J, Patrick DL, Williams B, Walwick J, Patrick MB (2006) The Rapid Assessment of Physical Activity (RAPA) among older adults. Prev Chronic Dis 3:A118

Trichopoulou A et al (1995) Diet and overall survival in elderly people. BMJ Clin Res Ed 311:1457-1460

Trichopoulou A, Costacou T, Bamia C, Trichopoulos D (2003) Adherence to a Mediterranean diet and survival in a Greek population. N Engl J Med 348:2599-2608. https://doi.org/10.1056/NEJMoa025039

Urbinati $S$ et al (2014) Secondary prevention after acute myocardial infarction: drug adherence, treatment goals, and predictors of health lifestyle habits. The BLITZ-4 registry. Eur J Prev Cardiol 22:1548 1556. https://doi.org/10.1177/2047487314561876

Vellone E, Pancani L, Greco A, Steca P, Riegel B (2016) Self-care confidence may be more important than cognition to influence self-care behaviors in adults with heart failure: testing a mediation model. Int J Nurs Stud 60:191-199. https://doi.org/10.1016/j. ijnurstu.2016.04.016

Veronesi G et al (2016) Educational class inequalities in the incidence of coronary heart disease in Europe. Heart 102:958. https://doi.org/10. 1136/heartjnl-2015-308909

Wójcicki K et al (2020) Knowledge and prevalence of risk factors for coronary artery disease in patients after the first and repeated percutaneous coronary intervention. Kardiol Pol 78:147-153. https://doi. org $/ 10.33963 / \mathrm{kp} .15070$

Yusuf S et al (2004) Effect of potentially modifiable risk factors associated with myocardial infarction in 52 countries (the INTERHEART study): case-control study. Lancet 364:937-952. https://doi.org/10. 1016/S0140-6736(04)17018-9

Publisher's note Springer Nature remains neutral with regard to jurisdictional claims in published maps and institutional affiliations. 